Results Eight-four (63\%) of the 133 were categorised, using the RFH-GA, as being moderately or severely malnourished. In contrast the MUST tool identified only 45 (34\%) patients as being at nutritional risk. Thus the sensitivity and specificity of MUST for determining nutritional risk were $34 \%$ (95\% CI 20 to 51) and $94 \%(95 \%$ CI 86 to 97); respectively; the $\kappa$ value was 0.19 demonstrating a poor level of agreement. The sensitivity and specificity of MUST improved when the patients with fluid retention were excluded from the analysis, $100 \%$ (95\% CI 46 to 100) and 91\% (74-98); respectively. The performance of the MUST also improved as the accurate dry body weight was better calculated but still did not reach $100 \%$ sensitivity indicating body weight alone is not a good marker of nutritional status in this patient population.

The performance of the MUST utilising alternative weight adjustments in patients with fluid retention

Conclusion The performance characteristics of the MUST tool in this setting are poor. This tool, can not be recommended for screening patients with chronic liver disease for nutritional risk.

Competing interests None declared.

\section{OC-076 ASSESSING KNOWLEDGE OF RECOGNITION AND TREATMENT OF MALNUTRITION AMONG HEALTHCARE PROFESSIONALS}

doi:10.1136/gutjn-2012-302514a.76

1J G Powell-Tuck, ${ }^{*}{ }^{2} \mathrm{~J}$ Connelly, ${ }^{3} \mathrm{H}$ Haboubi, ${ }^{1} \mathrm{P}$ Eadala. ${ }^{1}$ Department of Gastroenterology, Morriston Hospital, Swansea, UK; ${ }^{2}$ West Wales General Hospital, Carmarthen, UK; ${ }^{3}$ Department of Gastroenterology, Singleton Hospital, Swansea, UK

Introduction Malnutrition is common in hospitals, affecting up to $40 \%$ of patients. ${ }^{1}$ Malnourished patients are vulnerable to ill health and are known to be at increased risk of complications during inpatient stays. ${ }^{2}$ In 1999 Nightingale et al studied healthcare professionals' knowledge of nutrition and despite recognition of its importance, understanding was found to be poor. ${ }^{3}$ We aim to assess whether there has been any improvement in healthcare professionals' knowledge of nutrition.

Methods We approached healthcare staff from a tertiary referral centre and two district general hospitals to complete questionnaires to evaluate their understanding of assessment and treatment of under nutrition. The questionnaire contained 18 multiple choice questions in which staff were asked to select the correct answer from five possibilities. All questions were constructed from the 2006 NICE guidelines: Nutrition Support in Adults. ${ }^{2}$

Results We obtained 114 responses from 67 doctors, 10 nurses, 12 pharmacists, 10 dietitians and 15 final year medical students. Dietitians' average score was significantly higher than all other groups (81.7\%, $\mathrm{p}<0.001)$. Medical students scored lowest (25.4\%). Pharmacists averaged $42.6 \%$, doctors $35.8 \%$ and nurses $25.4 \%$. There was no statistically significant difference between medical and surgical specialties $(36.6 \%$ vs $33.6 \%, p=0.4)$. Consultants and registrars both averaged $43.8 \%$, SHOs $32.4 \%$ and $\mathrm{F} 1 \mathrm{~s} 28.8 \%$. Only $8.9 \%$ of doctors felt that they had received adequate nutritional training. $90.0 \%$ of nurses and $30.0 \%$ of doctors surveyed did not know how to calculate body mass index (BMI). Only $34.8 \%$ of doctors could correctly identify the BMI below which one would be considered underweight. $50.0 \%$ of doctors did not know an average person's daily fluid requirements. Knowledge of parenteral feeding and the nutritional needs of septic patients was also particularly poor among all health professionals except dietitians.

Conclusion These results suggest that basic understanding of nutrition remains poor. There needs to be greater emphasis on both undergraduate and postgraduate training in nutrition to ensure that recognition and treatment of malnutrition can be improved.

\section{Competing interests None declared.}

\section{REFERENCES}

1. Barker $\mathbf{L}$, et al. Hospital malnutrition: prevalence, identification and impact on patients and the healthcare system. Int J Environ Res Public Health 2011; 8:514-27

2. National Collaborating Centre for Acute Care. Nutritional Support in Adults, Oral Nutritional Support, Enteral Tube Feeding and Parenteral Nutrition. London: National Collaborating Centre for Acute Care, 2006.

3. Nightingale $\mathbf{J}$, Reeves $\mathrm{J}$. Knowledge about the assessment and management of undernutrition: a pilot questionnaire in a UK teaching hospital. Clin Nutr 1999; 18:23-7.

\section{OC-077 JEJUNAL TUBE FEEDING EXPERIENCE IN PAEDIATRIC NUTRITION SUPPORT}

doi:10.1136/gutjnl-2012-302514a.77

${ }^{1} \mathrm{C}$ E Paxton, ${ }^{*} \mathrm{P}$ M Gillett, ${ }^{2} \mathrm{G}$ Wilkinson, ${ }^{3} \mathrm{~F} \mathrm{D}$ Munro, ${ }^{2} \mathrm{~S}$ McGurk, ${ }^{1} \mathrm{~K}$ Armstrong ${ }^{1} \mathrm{~L}$ Bremner, ${ }^{1} \mathrm{~V}$ Robb, ${ }^{1} \mathrm{~J} \mathrm{E}$ Livingstone, ${ }^{1} \mathrm{D}$ A Devadason, ${ }^{1} \mathrm{D} \mathrm{J}$ Mitchell, ${ }^{4} \mathrm{D} \mathrm{C}$ Wilson. ${ }^{1}$ Department of Paediatric Gastroenterology and Nutrition, NHS Lothian, UK ${ }^{2}$ Department of Paediatric Radiology, NHS Lothian, UK; ${ }^{3}$ Department of Paediatric Surgery, NHS Lothian, UK; ${ }^{4}$ Child Life and Health, University of Edinburgh, Edinburgh, UK

Introduction There is an emerging group of children in whom poor and worsening upper GI dysmotility limits feed toleration and impacts growth; we wished to evaluate the role of jejunal tube feeding (JTF) in this group.

Methods A retrospective cohort study (database/clinical note review) in a tertiary paediatric centre to evaluate use of PEG-J, transgastric gastrojejunostomy (GJ) tubes and surgical roux-en $Y$ jejunostomy (ReYJ), and the impact on growth of JTF in children with worsening GI dysmotility. All children ( $<18$ years) receiving home enteral tube feeding (HETF) during the period 01 January 2002-31 December 2011. Weight at time of commencing JTF and at 6 or 12 months post-start was collected and expressed as SD or Z-score. Change in weight Z-score was calculated using paired t-test.

Results A total of 866 children received HETF during the study period, of whom $41(5 \%)$ had JTF at home. Median (range) decimal age at start of JTF was $2.7(0.1-16.2)$ years. 36 of 41 (88\%) had an underlying neurodisability; 33 of 41 (80\%) were gastrostomy fed prior to commencing JTF. Of the 41 JTF children, 19 (46\%) were fed via a GJ tube, 5 (12\%) via PEG-J and 17 (42\%) had a ReYJ. The majority of JTF related complications occurred with GJ tubes; although usually minor, one death occurred following small bowel intussusception around a GJ tube. Minor JTF complications included burst balloons, holes in the Y-port or tube and fungal infection and resolution required tube changes. Tube migration was a problem with both GJ and PEG-J tubes; ReYJ were associated with the fewest minor complications of stomal infection and leakage. By study end, 21 (51\%) continue on JTF, 9 (22\%) died (all but 1 due to their underlying condition), 1 (2\%) moved out of area, $2(5 \%)$ transitioned to adult services and $8(20 \%)$ returned to gastric feeding. 25 of 41 children had JTF for $>6$ months and had longitudinal growth data collected; median (range) weight Z-score at the start of JTF was $-1.3(-5.2-2.1)$ and rose to -1.0 (-3.4-2.3) by $6-12$ months, with a significant improvement in mean $(95 \% \mathrm{CI})$ change in weight Z-score of 0.7 (0.1 to 1.3$)$ $(\mathrm{p}=0.02)$.

Conclusion There are time consuming practical challenges associated with JTF, some of which are device dependent, and ReY JTF appears best for long-term usage. JTF is an effective intervention to improve growth in children with severe and worsening upper GI dysmotility.

Competing interests None declared. 